\title{
Millimeter- and submillimeter-wave spectrum of methyleneaminoacetonitrile ${ }^{\star}$
}

\author{
R. A. Motiyenko ${ }^{1}$, L. Margulès ${ }^{1}$, and J.-C. Guillemin ${ }^{2}$ \\ ${ }^{1}$ Laboratoire de Physique des Lasers, Atomes, et Molécules, UMR CNRS 8523, Université de Lille 1, \\ 59655 Villeneuve d'Ascq Cédex, France \\ e-mail: roman.motienko@univ-lille1.fr \\ 2 École Nationale Supérieure de Chimie de Rennes, CNRS, UMR 6226, 11 allée de Beaulieu, CS 50837, 35708 Rennes Cedex 7, \\ France
}

Received 26 July 2013 / Accepted 11 September 2013

\section{ABSTRACT}

\begin{abstract}
Context. Aminoacetonitrile has been detected in the interstellar medium, and the Strecker-type synthesis is considered as one of its possible formation mechanisms in this medium. Methyleneaminoacetonitrile $\left(\mathrm{CH}_{2} \mathrm{NCH}_{2} \mathrm{CN}\right.$, MAAN) is one of the by-products of the Strecker reaction and a good candidate for astrophysical detection.

Aims. The rotational spectrum of MAAN has never been studied before. To provide the basis for MAAN detection in the interstellar medium we studied its millimeter- and submillimeter-wave spectrum.

Methods. The rotational spectrum of MAAN was measured in the frequency range of 120-600 GHz. The spectroscopic study was supported by theoretical calculations of the molecular structure and harmonic force field.

Results. The ground- and the two lowest excited vibrational states of the most stable synperiplanar conformation of MAAN were assigned and analyzed. The obtained sets of rotational constants allow us to accurately predict the transition frequencies of MAAN in the frequency range up to $900 \mathrm{GHz}$.
\end{abstract}

Key words. ISM: molecules - methods: laboratory: molecular - submillimeter: ISM - molecular data - line: identification

\section{Introduction}

Alpha-aminonitriles are commonly prepared by a Strecker reaction starting from an aldehyde, ammonia, and hydrogen cyanide (see Fig. 1). Their acidic hydrolysis leads to the corresponding $\alpha$-amino acids (Kendall \& McKenzie 1941). This two-step sequence has often been proposed as one of the most important reactions that occurred on primitive Earth for the synthesis of many amino acids, the building blocks of life. The quite recent observation in the interstellar medium of the simplest $\alpha$-aminonitrile, the aminoacetonitrile $\mathrm{NCCH}_{2} \mathrm{NH}_{2}$ (Belloche et al. 2008), generated several investigations on the possibility of its formation in this medium via a Strecker reaction starting from the simplest aldehyde, the formaldehyde (Danger et al. 2011). In laboratory conditions, the aminoacetonitrile easily gives the methylene aminoacetonitrile (MAAN) in the presence of an excess of formaldehyde (Adam \& Langley 1956). In a natural medium such as the interstellar medium, it is unrealistic to expect stoechiometric amounts of reagents and this compound as well as other by-products such as hydroxyacetonitrile (in the absence of ammonia; Danger et al. 2013) or methanimine and then hexamethylenetetramine (in the absence of hydrogen cyanide; Vinogradoff et al. 2012) might be formed. Therefore, the possible role of MAAN in prebiotic chemistry on primitive Earth has been proposed as well several decades ago (Choughuley et al. 1972), but has received a mediocre response even though if it has been described as an efficient substrate in the preparation of peptides (Subbaraman et al. 1975). However, while the

^ Full Table 2 is available at the CDS via anonymous ftp to cdsarc.u-strasbg.fr (130.79.128.5) or via http://cdsarc.u-strasbg.fr/viz-bin/qcat?]/A+A/559/A44 aminoacetonitrile decomposes in a few hours at room temperature, MAAN forms a stable trimer that can restore the monomer on heating (Dammel \& Bock 1987). As for the aminoacetonitrile, the hydrolysis of MAAN gives glycine, and the trimer can be considered as a stable precursor of this compound. On the other hand, with only one atom more than aminoacetonitrile, the MAAN appears to be a serious candidate for the interstellar medium. To promote its astrophysical detection we report here our study of the rotational spectrum of MAAN in the frequency range of $120-600 \mathrm{GHz}$.

\section{Experiments}

The commercially available trimer $(1 \mathrm{~g})$ was introduced in a flask connected to a vacuum line. The flask was degassed and then heated with a heat gun to $300{ }^{\circ} \mathrm{C}$ to obtain the expected vapor pressure of the monomer (yield $>90 \%$ ). The microwave analysis was directly performed by analysis of the gaseous flow. Condensation of the product on a cold finger cooled at $77 \mathrm{~K}$ led to the oligomerization on heating before the revaporization of the MAAN. In the literature, the trimer was often used considered as the monomer. The same error is still observed for some commercially available samples. MAAN is a kinetically very unstable compound at room temperature. $\tau_{1 / 2}\left(5 \%\right.$ in $\left.\mathrm{CDCl}_{3}, 25^{\circ} \mathrm{C}\right)$ : 20 min. $\tau_{1 / 2}\left(5 \%\right.$ in $\left.\mathrm{CD}_{2} \mathrm{Cl}_{2}, 25^{\circ} \mathrm{C}\right): 2$ h. The results of the nuclear magnetic resonance (NMR) spectroscopy of MAAN are given in Appendix A.

The measurements of the rotational spectrum of MAAN were performed in the frequency range of $120-180 \mathrm{GHz}$ using the Lille backward wave oscillator based fast-scan spectrometer (Alekseev et al. 2012), and in the frequency range 


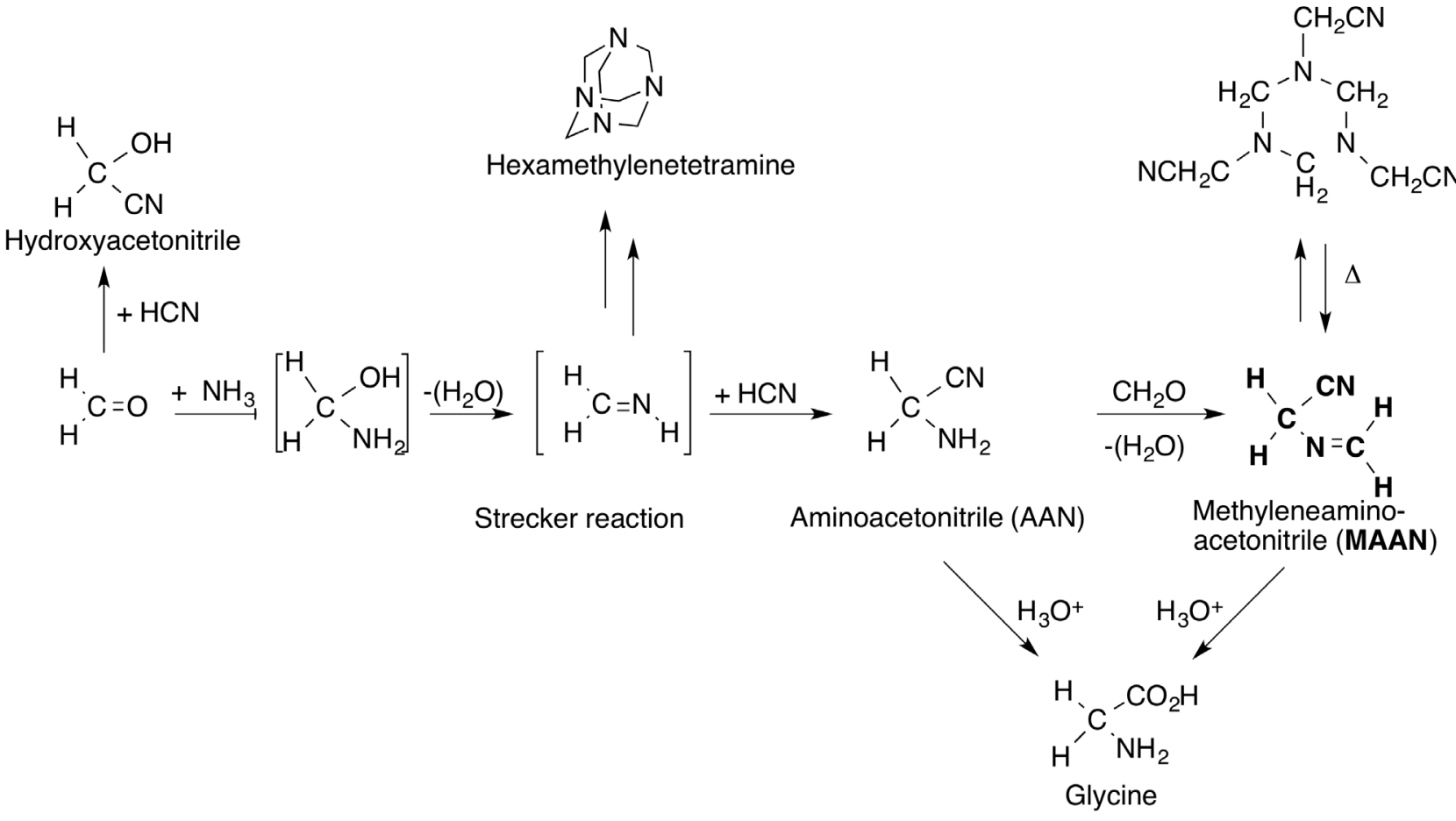

Fig. 1. Synthesis of MAAN and various other compounds formed starting from formaldehyde, ammonia, and hydrogen cyanide as a function of their abundance.

\section{a) synperiplanar}

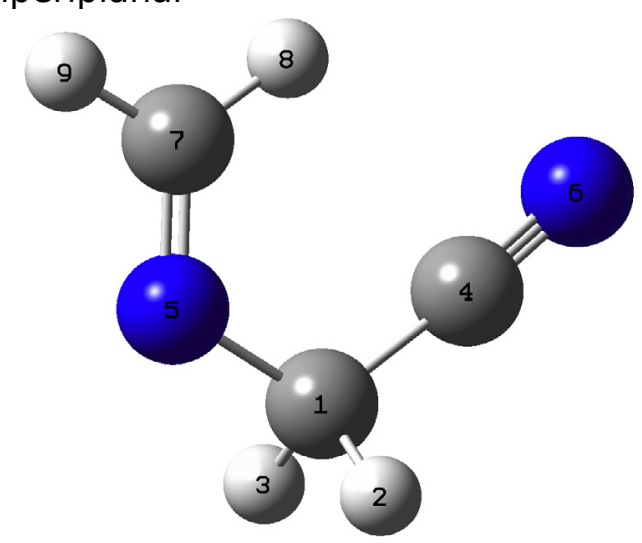

b) anticlinal

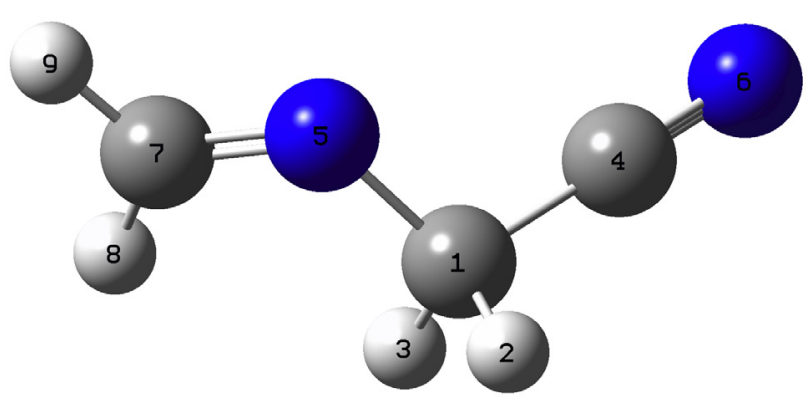

Fig. 2. Structure and atom numbering for $s p$ a) and $a c$ b) conformations of MAAN.

of $180-600 \mathrm{GHz}$ using a spectrometer based on solid-state sources (Motiyenko et al. 2010). The experiment was performed in a static mode, when the stainless-steel absorption cell was filled with the MAAN sample at an optimum pressure of about $20 \mu$ bar. Owing to the kinetic instability of the sample, the absorption cell was refilled after $4-5 \mathrm{~h}$ to minimize the spectral features of various decomposition products.

\section{Quantum chemical calculations}

The spectroscopic work was supported by quantum chemical calculations to provide an initial basis for spectral assignments. The ab initio and density functional theory (DFT) calculations were performed by employing the Gaussian 09 suite of programs (Frisch et al. 2009). Calculations were performed using Møller-Plesset second-order perturbation calculations (MP2; Møller \& Plesset 1934), and density functional theory (DFT) calculations employing Becke's three-parameter hybrid functional (Becke 1988) and the Lee, Yang and Parr correlation functional (B3LYP; Lee et al. 1988). The Peterson and Dunning's (Peterson $\&$ Dunning 2002) correlation-consistent triple- $\zeta$ wave function augmented with diffuse function aug-cc-pVTZ was employed in the MP2 calculations. The $6-311++\mathrm{G}(3 \mathrm{df}, 2 \mathrm{pd})$ wave function augmented with diffuse functions was employed in the B3LYP calculations.

The results of the calculations have shown two stable conformations of MAAN that differ by the value of the $\tau\left(\mathrm{C}_{4}-\mathrm{C}_{1}-\mathrm{N}_{5}-\right.$ $\left.\mathrm{C}_{7}\right)$ dihedral angle. The most stable synperiplanar $\left(s p, \tau=0^{\circ}\right)$ conformation is characterized by the plane of symmetry created by all heavy atoms. The anticlinal $\left(a c, \tau=120^{\circ}\right)$ conformation possesses no symmetry and is less energetically favorable. The energy difference between two conformations is $770 \mathrm{~cm}^{-1}$ (MP2/aug-cc-pVTZ calculations). The $a c$ conformation has two structurally equivalent minima on the potential curve that characterizes the $\tau$ dihedral angle $\left(\tau= \pm 120^{\circ}\right)$. According to the results of the scan of the potential energy surface determined by $\tau$, the interconversion barrier between the $s p$ and $a c$ conformations is about $1100 \mathrm{~cm}^{-1}$ (MP2/6-311++G(3df, 2pd) calculations).

In addition to structural optimizations, we calculated harmonic force field parameters to provide the information on 
Table 1. Rotational constants of MAAN.

\begin{tabular}{|c|c|c|c|c|}
\hline Parameters & Ground state & Theory & $v_{21}=1$ & $v_{20}=1$ \\
\hline$A(\mathrm{MHz})$ & $12318.41521(26)^{a}$ & 12123.12 & $12331.380(13)$ & $12386.024(13)$ \\
\hline$B(\mathrm{MHz})$ & $3912.427270(66)$ & 3979.06 & $3903.4857(22)$ & $3912.4129(22)$ \\
\hline$C(\mathrm{MHz})$ & $3022.364639(55)$ & 3051.76 & $3023.676651(91)$ & $3020.55463(11)$ \\
\hline$\Delta_{J}(\mathrm{kHz})$ & $3.069842(21)$ & 3.405 & $3.08068(10)$ & 2.99944(11) \\
\hline$\Delta_{J K}(\mathrm{kHz})$ & $-19.87015(13)$ & -23.45 & $-20.3285(19)$ & $-19.0813(20)$ \\
\hline$\Delta_{K}(\mathrm{kHz})$ & $64.21747(88)$ & 67.60 & $64.351(25)$ & 64.832(30) \\
\hline$\delta_{J}(\mathrm{kHz})$ & $1.007889(15)$ & 1.146 & $1.009530(64)$ & $0.980315(68)$ \\
\hline$\delta_{K}(\mathrm{kHz})$ & $5.51296(10)$ & 4.882 & $5.5382(16)$ & $5.8723(17)$ \\
\hline$\Phi_{J}(\mathrm{~Hz})$ & $0.0086602(44)$ & & $0.0083710(70)$ & $0.0075894(76)$ \\
\hline$\Phi_{J K}(\mathrm{~Hz})$ & $-0.038544(54)$ & & $-0.03642(34)$ & $-0.02510(33)$ \\
\hline$\Phi_{K J}(\mathrm{~Hz})$ & $-0.19434(28)$ & & $-0.2325(34)$ & $-0.2107(36)$ \\
\hline$\Phi_{K}(\mathrm{~Hz})$ & 1.04940(94) & & {$[1.0494]^{b}$} & {$[1.0494]^{b}$} \\
\hline$\phi_{J}(\mathrm{~Hz})$ & $0.0041171(29)$ & & $0.0039678(45)$ & $0.0036143(49)$ \\
\hline$\phi_{K}(\mathrm{~Hz})$ & $0.64226(58)$ & & $0.6500(43)$ & $0.7160(47)$ \\
\hline$L_{J}(\mathrm{mHz})$ & $-0.00003323(29)$ & & {$[0.0]$} & {$[0.0]$} \\
\hline$L_{J J K}(\mathrm{mHz})$ & $0.0001352(33)$ & & {$[0.0]$} & {$[0.0]$} \\
\hline$L_{J K}(\mathrm{mHz})$ & $-0.001548(27)$ & & {$[0.0]$} & {$[0.0]$} \\
\hline$L_{K K J}(\mathrm{mHz})$ & $0.00649(16)$ & & {$[0.0]$} & {$[0.0]$} \\
\hline$l_{J}(\mathrm{mHz})$ & $-0.00001690(18)$ & & {$[0.0]$} & {$[0.0]$} \\
\hline$\Delta E(\mathrm{MHz})$ & & & \multicolumn{2}{|c|}{$454842.08(12)$} \\
\hline$G_{a}(\mathrm{MHz})$ & & & \multicolumn{2}{|c|}{$6534.61(45)$} \\
\hline$G_{a}^{J}(\mathrm{MHz})$ & & & \multicolumn{2}{|c|}{$0.008065(36)$} \\
\hline$G_{a}^{K}(\mathrm{MHz})$ & & & \multicolumn{2}{|c|}{$-0.11558(69)$} \\
\hline$G_{b}(\mathrm{MHz})$ & & & \multicolumn{2}{|c|}{ 2447.68(21) } \\
\hline$G_{b}^{J}(\mathrm{MHz})$ & & & \multicolumn{2}{|c|}{$-0.001409(19)$} \\
\hline$G_{b}^{K}(\mathrm{MHz})$ & & & \multicolumn{2}{|c|}{$0.12014(59)$} \\
\hline$G_{b}^{J K}(\mathrm{kHz})$ & & & \multicolumn{2}{|c|}{$-0.000953(52)$} \\
\hline $\mathrm{N}^{c}$ & 1684 & & 540 & 551 \\
\hline$\sigma(\mathrm{MHz})^{d}$ & 0.029 & & 0.021 & 0.022 \\
\hline$\sigma_{w}^{e}$ & 0.64 & & 0.55 & 0.55 \\
\hline
\end{tabular}

Notes. ${ }^{(a)}$ Number in parentheses are one time the standard deviation. ${ }^{(b)}$ Fixed to the ground state value. ${ }^{(c)}$ Number of distinct frequency lines in fit. ${ }^{(d)}$ Standard deviation of the fit. ${ }^{(e)}$ Weighted deviation of fit.

quartic centrifugal distortion constants as well as on lowfrequency vibrational modes. This information is very useful especially at the initial stage of spectral assignment in our case, which usually starts with relatively high values of the $J$ quantum number and where centrifugal distortion corrections provide much more realistic theoretical spectra. The results of the quantum chemical calculations are given in Tables B.1-B.4.

\section{Analysis and fit}

Owing to the low Boltzmann factor 0.025 for the $a c$ conformer, we did not expect to observe any of its spectral features even at room temperature. Therefore we concentrated our effort on assigning of the most stable $s p$ conformer. It is a prolate asymmetric top (Ray's asymmetry parameter $\kappa=-0.81$ ) with two non-zero dipole moment components determined from ab initio calculations, $\mu_{a}=1.8 \mathrm{D}$ and $\mu_{b}=0.8 \mathrm{D}$.

The assignment started from the search for, as expected, the strongest ${ }^{a} R_{0,1}$ transitions with $K_{a}=0$ and 1 and $K_{c}=J$, accompanied by much less intense ${ }^{b} R_{ \pm 1,1}$ transitions with the same $K_{a}$ and $K_{c}$. For the $s p$ conformer of MAAN for $J=17 \ldots 23$ these transitions form easily distinguishable quartets with splittings that reduce with $J$ increasing. Starting from $J=24$ and for higher values of $J$, such a quartet is represented by a single line in our spectra. Six transition quartets were easily located and assigned and produced the first experimental set of rotational parameters containing $B$ and $C$ constants as well as the $\Delta_{J}$ centrifugal correction term. The frequency predictions calculated on the basis of the experimental rotational parameters allowed the assignment of the next series of quartets with the same selection rules and $K_{a}=1$ and 2 , and $K_{c}=J-1$. In this way, the $A$ rotational constants and $\delta_{J}$ and $\delta_{K}$ centrifugal distortion terms were determined. The following assignment was carried out in bootstrap manner, for which newly assigned transitions were used to improve the values of rotational parameters and to provide new more precise frequency predictions that were used for new assignments. In total, about 1500 distinct frequency lines were assigned to the ground vibrational state of MAAN. The highest values of the $J$ and $K_{a}$ quantum numbers for the assigned transitions of the ground state are 97 and 41. Most of the lines are $a$-type transitions and only about $20 \%$ are $b$-type transitions which were found to be generally much weaker than $a$-type lines. All the assigned ground state rotational transitions of MAAN were fitted within experimental accuracy to a standard Watson A-reduction Hamiltonian (Watson 1977) in $I^{r}$ coordinate representation. The set of rotational and centrifugal distortion constants is presented in Table 1 . The rms deviation of the fit has a reasonable value of $0.029 \mathrm{MHz}$, but a somewhat better result 
Table 2. A part of the table available at the CDS, with assigned rotational transitions of MAAN.

\begin{tabular}{cccccccccccc}
\hline \hline$J^{\prime \prime}$ & $K_{a}^{\prime \prime}$ & $K_{c}^{\prime \prime}$ & $v^{\prime \prime}$ & $J^{\prime}$ & $K_{a}^{\prime}$ & $K_{c}^{\prime}$ & $v^{\prime}$ & $\begin{array}{c}\text { Measured frequency } \\
(\mathrm{MHz})\end{array}$ & $\begin{array}{c}\text { Residual } \\
(\mathrm{MHz})\end{array}$ & $\begin{array}{c}\text { Uncertainty } \\
(\mathrm{MHz})\end{array}$ & $\begin{array}{c}\text { Weighted relative } \\
\text { intensity }\end{array}$ \\
\hline 38 & 14 & 25 & $2^{a}$ & 37 & 14 & 24 & 2 & 266918.5150 & 0.0146 & 0.030 & 0.50 \\
38 & 14 & 24 & 2 & 37 & 14 & 23 & 2 & 266918.5150 & 0.0146 & 0.030 & 0.50 \\
38 & 12 & 27 & 1 & 37 & 12 & 26 & 1 & 266923.9450 & -0.0011 & 0.030 & \\
38 & 7 & 32 & 0 & 37 & 7 & 31 & 0 & 266977.3390 & 0.0030 & 0.030 & \\
38 & 12 & 26 & 1 & 37 & 12 & 25 & 1 & 267117.5740 & 0.0050 & 0.030 & \\
40 & 5 & 36 & 1 & 39 & 5 & 35 & 1 & 267130.3020 & -0.0200 & 0.050 & 0.50 \\
38 & 13 & 26 & 0 & 37 & 13 & 25 & 0 & 267190.0770 & 0.0130 & 0.030 & 0.50 \\
38 & 13 & 25 & 0 & 37 & 13 & 24 & 0 & 267190.0770 & 0.0130 & 0.030 & \\
41 & 4 & 38 & 1 & 40 & 4 & 37 & 1 & 267321.9540 & -0.0185 & 0.050 & \\
41 & 3 & 38 & 1 & 40 & 3 & 37 & 1 & 267323.3110 & -0.0039 & 0.050 & \\
40 & 5 & 36 & 0 & 39 & 5 & 35 & 0 & 267621.5020 & -0.0110 & 0.030 & \\
\hline
\end{tabular}

Notes. ${ }^{(a)}$ Vibrational assignment: $0-$ ground state, $1-v_{21}=1$ excited state, $2-v_{20}=1$ excited state.

Table 3. Rotational and vibrational partition functions at various temperatures for the sp conformation of MAAN.

\begin{tabular}{ccc}
\hline \hline Temperature $(\mathrm{K})$ & $Q(T)_{\text {rot }}$ & $Q(T)_{\text {vib }}$ \\
\hline 300 & 72581.26 & 5.463 \\
200 & 39508.23 & 2.437 \\
150 & 25661.35 & 1.686 \\
50 & 4938.53 & 1.018 \\
10 & 441.72 & 1.000 \\
\hline
\end{tabular}

of $0.021 \mathrm{MHz}$ can be obtained when the $l_{K}$ octic centrifugal distortion constant is included in the model. However, it also leads to a high correlation between the $L_{J K}, L_{K K J}$, and $l_{K}$ parameters and to a poor conditioning of the problem. Therefore, in the final fit the $l_{K}$ parameter was not taken into account.

In addition to ground state lines we assigned in the observed spectra the features belonging to the lowest excited vibrational states. According to the quantum chemical calculations for the $s p$ conformer of MAAN, there are two low-frequency vibrational modes: $v_{21}$, which corresponds to torsional motion and is characterized by the variation of the dihedral angle $\tau$ defined previously, and in-plane bending vibration $v_{20}$, which is characterized by variation of the angle $\alpha\left(\mathrm{C}_{4}-\mathrm{C}_{1}-\mathrm{N}_{5}\right)$. The corresponding vibrational frequencies of the modes are $152 \mathrm{~cm}^{-1}$ and $154 \mathrm{~cm}^{-1}$, and therefore one might expect a Coriolis-type interaction between them. Indeed, at first we were able to assign the lines belonging only to the $v_{21}$ state. They were distinguishable by quartet patterns for low $K_{a}$ values similar to the ground state lines. While the intensities of the rotational lines of the $v_{20}$ were expected to be the same as for $v_{21}$ state, we were unable to assign any similar pattern at least for low $K_{a}$ transitions of $v_{20}$ state at first. The only transitions of the $v_{20}$ state we were able to assign were found in the high-frequency part of the spectra and corresponded to high $J$ quantum number values. Another indicator of the interaction between two states were the anomalous values of the centrifugal distortion constants obtained for the $v_{21}$ state in a single-state fit. Typically, vibrational excitation leads to relatively small variations of rotational and centrifugal distortion constants that do not exceed $100 \%$, whereas for the $v_{21}$ state the $\Delta_{K}$ and $\delta_{K}$ centrifugal distortion constants differed from the ground state ones by a factor of 10 or more. All the assigned transitions of the coupled states were fitted using the following standard model:

$\mathbf{H}=\left(\begin{array}{cc}H_{\text {rot }}^{(21)} & H_{\mathrm{cor}} \\ H_{\mathrm{cor}} & H_{\mathrm{rot}}^{(20)}+\Delta E\end{array}\right)$, where $H_{\text {rot }}^{(20)}$ and $H_{\text {rot }}^{(21)}$ are the standard rotational Watson A-reduction Hamiltonians, $\Delta E$ is the energy difference between two coupled states, and $H_{\text {cor }}$ is the off-diagonal Coriolis interaction term.

The equilibrium configuration of the $s p$ conformer of MAAN is described by the $C_{s}$ point group. In-plane bending and out-ofplane torsion belong to the $A^{\prime}$ and $A^{\prime \prime}$ irreducible representations of the group. In this case, Coriolis interaction is allowed along the $a$ and $b$ axes, and the Hamiltonian $H_{\text {cor }}$ used in the present study is expressed as

$H_{\mathrm{cor}}=i\left(G_{a}+G_{a}^{J} P^{2}+G_{a}^{K} P_{z}^{2}+\ldots\right) P_{z}+$
$i\left(G_{b}+G_{b}^{J} P^{2}+G_{b}^{K} P_{z}^{2}+G_{b}^{J K} P^{2} P_{z}^{2} \ldots\right) P_{x}$

where $G_{a}$ and $G_{b}$ are the Coriolis coupling constants, and all other parameters are their respective centrifugal distortion corrections.

For the initial fit using the Hamiltonian (1), we fixed all centrifugal distortion constants in $H_{\text {rot }}$ at values of the ground state ones. We also kept fixed the energy difference between two interacting states and let only vary the $G_{a}$ constant. Starting from the value of the energy difference between two vibrational modes obtained from ab initio calculations $\left(1.8 \mathrm{~cm}^{-1}\right)$, we searched for the best-fitting solution by manually changing $\Delta E$ with a step of $0.003 \mathrm{~cm}^{-1}$ and keeping it fixed in the fit. The best-fit solution was found for the value of $\Delta E \simeq 15 \mathrm{~cm}^{-1}$. The consistency of the parameters produced by the first best fit was checked by predicting and assigning new series of highly perturbed transitions of both interacting states. The following assignment did not represent any major difficulty. In total about 1000 rotational transitions of $v_{21}$ and $v_{20}$ states were assigned and fitted within the experimental accuracy. The energy difference determined $\Delta E=15.1718987(40) \mathrm{cm}^{-1}$ is much higher than the theoretical value, but still within the error of the quantum chemical calculations.

The results of the global fit using the Hamiltonian (1) are presented in Table 1. The complete list of measured rotational transitions of the ground, $v_{20}$, and $v_{21}$ states is presented in Table 2. Here only a part of Table 2 is shown as an example.

We also provide the partition function for MAAN given in Table 3 with $Q(T)_{\text {tot }}=Q(T)_{\text {rot }} Q(T)_{\text {vib }}$ for different temperatures. Simple formulas for calculating $Q(T)_{\text {rot }}$ and $Q(T)_{\text {vib }}$ can be found elsewhere (see for example Haykal et al. 2013). The experimental rotational constants and the calculated harmonic frequencies were used. 


\section{Conclusions}

The spectroscopic information we obtained is expected to be enough to provide the basis for astrophysical detection of MAAN. The present analysis of the ground and the two lowest excited vibrational states covers the frequency range of 150$600 \mathrm{GHz}$ where the most intense transitions are expected at low temperatures. It allows obtaining accurate frequency predictions at least for transitions involving levels with $J \leq 90$ and $K_{a} \leq 30$ and in the frequency range up to $900 \mathrm{GHz}$. We resolved no nuclear quadrupole hyperfine structure in the present study, mostly owing to the Doppler-limited resolution of the spectrometers. However, in the $\mathrm{cm}$-wave range for transitions involving low $J$ and $K_{a}$ values the hyperfine structure can be resolved even at Doppler resolution. For this purpose we also provide in the Table B.4 the values of elements of nuclear quadrupole coupling tensors for both nitrogen nuclei. These theoretical values in combination with accurate rotational parameters are expected to result in reliable predictions on the hyperfine splittings.

Acknowledgements. This work was supported by the Programme National "Physique et Chimie du Milieu Interstellaire" (INSU-CNRS) and the Centre National d'Études Spatiales (CNES). Gregoire Danger (Université de Provence, Marseille, France) is acknowledged for helpful discussions.

\section{Appendix A: NMR of MAAN}

Nuclear magnetic resonance (NMR) spectroscopy allows one to quickly characterize the expected compound. This technique confirms the monomeric structure of the vaporized product, mainly by the chemical shifts in ${ }^{1} \mathrm{H}$ and ${ }^{13} \mathrm{C}$ NMR of the $\mathrm{H}_{2} \mathrm{C}=\mathrm{N}$ group, which is substantially different from those of the cyclic $\left(-\mathrm{H}_{2} \mathrm{C}-\mathrm{N}-\right)_{3}$ ring of the commercially available trimer precursor (see the section "Experiments" at the end of the first paragraph).

${ }^{1} \mathrm{H} \mathrm{NMR}\left(\mathrm{CDCl}_{3}, 400 \mathrm{MHz}, 25{ }^{\circ} \mathrm{C}\right) \delta 4.53\left(t, 2 \mathrm{H},{ }^{4} J_{\mathrm{HH}}=\right.$ $\left.2.4 \mathrm{~Hz}, \mathrm{CH}_{2}-\mathrm{CN}\right) ; 7.42\left(1 \mathrm{H}\right.$, part $\mathrm{A}$ of $\mathrm{AB}$ system, ${ }^{2} J_{\mathrm{HH}}=$ $\left.14.5 \mathrm{~Hz},{ }^{4} J_{\mathrm{HH}}=2.4 \mathrm{~Hz}, \mathrm{~N}=\mathrm{CHH}\right) ; 7.64(1 \mathrm{H}, \mathrm{B}$ part of an $\mathrm{AB}$ system, $\left.{ }^{2} J_{\mathrm{HH}}=14.5 \mathrm{~Hz},{ }^{4} J_{\mathrm{HH}}=2.4 \mathrm{~Hz}, \mathrm{~N}=\mathrm{CHH}\right)$. ${ }^{13} \mathrm{C} \mathrm{NMR}\left(\mathrm{CDCl}_{3}, 100 \mathrm{MHz}, 25^{\circ} \mathrm{C}\right) \delta 46.7\left({ }^{1} J_{\mathrm{CH}}=147.4 \mathrm{~Hz}(\mathrm{t})\right.$, $\left.\mathrm{CH}_{2} \mathrm{CN}\right) ; 114.4(\mathrm{~s}, \mathrm{CN}), 157.0\left({ }^{1} J_{\mathrm{CH}}=161.4 \mathrm{~Hz}(\mathrm{~d}),{ }^{1} J_{\mathrm{CH}}=\right.$ $181.9 \mathrm{~Hz}(\mathrm{~d}) \mathrm{N}=\mathrm{CH}_{2}$ ).

\section{Appendix B: Results of the ab initio calculations on MAAN}

Table B.1. The calculated MP2/aug-cc-pVTZ molecular structure (Z-matrix) of the $s p$ conformation of MAAN.

\begin{tabular}{ccccccc}
\hline \hline Atom & NA & NB & NC & $\begin{array}{c}\text { Bond } \\
\text { length (pm) }\end{array}$ & $\begin{array}{c}\text { Angle } \\
\circ\end{array}$ & $\begin{array}{c}\text { Dihedral } \\
\circ\end{array}$ \\
\hline $\mathrm{C}$ & & & & 146.70 & & \\
$\mathrm{C}$ & 1 & & & 109.00 & 109.05 & \\
$\mathrm{H}$ & 2 & 1 & & 109.00 & 109.05 & 116.53 \\
$\mathrm{H}$ & 2 & 1 & 3 & 145.90 & 115.67 & -121.73 \\
$\mathrm{~N}$ & 2 & 1 & 3 & 127.42 & 118.86 & 0.00 \\
$\mathrm{C}$ & 5 & 2 & 1 & 12.13 & 175.84 & 0.00 \\
$\mathrm{~N}$ & 1 & 2 & 5 & 117.13 & 117.59 & -180.00 \\
$\mathrm{H}$ & 6 & 5 & 2 & 108.38 & 112.05 & 0.00 \\
$\mathrm{H}$ & 6 & 5 & 2 & 109.21 & 124.05 & \\
\hline
\end{tabular}

Table B.2. The calculated MP2/aug-cc-pVTZ molecular structure (Z-matrix) of the $a c$ conformation of MAAN.

\begin{tabular}{ccccccc}
\hline \hline Atom & NA & NB & NC & $\begin{array}{c}\text { Bond } \\
\text { length }(\mathrm{pm})\end{array}$ & $\begin{array}{c}\text { Angle } \\
{ }^{\circ}\end{array}$ & $\begin{array}{c}\text { Dihedral } \\
{ }^{\circ}\end{array}$ \\
\hline $\mathrm{C}$ & & & & & & \\
$\mathrm{C}$ & 1 & & & 146.38 & & \\
$\mathrm{H}$ & 2 & 1 & & 109.66 & 108.70 & \\
$\mathrm{H}$ & 2 & 1 & 3 & 109.04 & 108.20 & 117.64 \\
$\mathrm{~N}$ & 2 & 1 & 4 & 145.89 & 109.91 & 118.13 \\
$\mathrm{C}$ & 5 & 2 & 1 & 127.39 & 115.50 & 119.98 \\
$\mathrm{~N}$ & 1 & 2 & 5 & 117.01 & 178.94 & -120.65 \\
$\mathrm{H}$ & 6 & 5 & 2 & 108.38 & 118.53 & 179.45 \\
$\mathrm{H}$ & 6 & 5 & 2 & 109.37 & 123.25 & -0.62 \\
\hline
\end{tabular}

Table B.3. MP2/aug-cc-pVTZ spectroscopic parameters of the $a c$ conformation of MAAN.

\begin{tabular}{lc}
\hline \hline Parameter & Value \\
\hline$A(\mathrm{MHz})$ & 20292.67 \\
$B(\mathrm{MHz})$ & 2836.63 \\
$C(\mathrm{MHz})$ & 2696.66 \\
$\Delta_{J}(\mathrm{kHz})$ & 2.779 \\
$\Delta_{J K}(\mathrm{kHz})$ & -117.6 \\
$\Delta_{K}(\mathrm{kHz})$ & 1634.5 \\
$\delta_{J}(\mathrm{kHz})$ & 0.471 \\
$\delta_{K}(\mathrm{kHz})$ & -2.114 \\
\hline
\end{tabular}

Table B.4. Elements of nuclear quadrupole coupling tensors (in $\mathrm{MHz}$ ) for the $s p$ and $a c$ conformations of MAAN.

\begin{tabular}{ccccc}
\hline \hline & \multicolumn{2}{c}{$s p$} & \multicolumn{2}{c}{$a c$} \\
& $N_{5}$ & $N_{7}$ & $N_{5}$ & $N_{7}$ \\
\hline$\chi_{a a}$ & -1.6948 & -1.8018 & 1.7372 & -2.6638 \\
$\chi_{b b}$ & -1.2432 & -0.0696 & 2.2026 & 0.7632 \\
$\chi_{c c}$ & 2.9380 & 1.8713 & -3.9397 & 1.9006 \\
$\chi_{a b}$ & -3.2191 & -2.9799 & 0.2459 & -2.4839 \\
$\chi_{a c}$ & 0.0 & 0.0 & -1.5499 & -0.0474 \\
$\chi_{b c}$ & 0.0 & 0.0 & -1.8088 & 0.1006 \\
\hline
\end{tabular}

\section{References}

Adam, R., \& Langley, W. D. 1956, Organic Syntheses Coll., 1, 355

Alekseev, E. A., Motiyenko, R. A., \& Margulès, L. 2012, Radio Phys. Radio Astron., 3, 75

Becke, A. D. 1988, Phys. Rev. A, 38, 3098

Belloche, A., Menten, K. M., Comito, C., et al. 2008, A\&A, 482, 179

Choughuley, A. S. U., Subbaraman, A. S., \& Kazi, Z. A. 1972, Ind. J. Biochem. Biophys., 9, 144

Dammel, R., \& Bock, H. 1987, Z. Naturforsch. B, 42, 810

Danger, G., Borget, F., Chomat, M., et al. 2011, A\&A, 535, A47

Danger, G., Duvernay, F., Borget, F., et al. 2013, A\&A, 549, A93

Frisch, M. J., Trucks, G. W., Schlegel, H. B., et al. 2009, Gaussian 09, revision A. 1 (Pittsburgh PA: Gaussian, Inc.)

Haykal, I., Motiyenko, R. A., Margulès, L., \& Huet, T. R. 2013, A\&A, 549, A96

Kendall, E. C., \& McKenzie, B. F. 1941, Organic Syntheses Coll., 1, 21

Lee, C., Yang, W., \& Parr, R. G. 1988, Phys. Rev. B, 37, 785

Møller, C., \& Plesset, M. S. 1934, Phys. Rev., 46, 618

Motiyenko, R. A., Margulès, L., Alekseev, E. A., Guillemin, J. C., \& Demaison, J., 2010, J. Mol. Spectr., 264, 94

Peterson, K. A., \& Dunning, T. H., Jr. 2002, J. Chem. Phys., 117, 10548

Subbaraman, A. S., Kazi, Z. A., Choughuley, A. S. U., \& Chadha, M. S. 1975, Origins of Life, 6, 537

Vinogradoff, V., Rimola, A., Duvernay, F., et al. 2012, Phys. Chem. Chem. Phys., 14,12309

Watson, J. K. G. 1977, Vibrational Spectra and Structure, 6 (Amsterdam: Elsevier), 1 